\title{
A Heavy Metal Path to New Physics
}

\section{Jan Hajer*}

Université catholique de Louvain

E-mail: jan.hajer@uclouvain.be

I present a proposal to search for long lived particles in heavy ion collisions using heavy neutrinos as an example. In comparison to proton collisions heavy ion collisions allow to lower the trigger thresholds significantly. On the other hand, there has been an interested to operate the LHC using ions lighter than lead. We have shown that searches in intermediate ion collisions can have a higher significance than in proton collision if one assumes equal running time.

XXVII International Workshop on Deep-Inelastic Scattering and Related Subjects - DIS2019 8-12 April, 2019

Torino, Italy

${ }^{*}$ Speaker. 


\section{Introduction}

Until now the LHC has not found New Physics beyond the SM. While the initial focus of the experimental effort lay on heavy New Physics, it will shift towards searches of weakly coupled particles during the high luminosity run. We have proposed to utilize also the heavy ion runs for this goal $[1,2]$.

\section{Heavy Ion collisions}

The main advantages of heavy ion collisions for New Physics searches are the absence of pile-up, which leads to a single primary vertex and, more importantly, the large nucleon multiplicity (e.g. $A(\mathrm{~Pb})=208$ and $Z(\mathrm{~Pb})=82$ in the case of lead), which results in a high number of parton level interactions per collision e.g. $\sigma_{\mathrm{PbPb}} / \sigma_{p p} \propto A^{2}=43 \cdot 10^{3}$. The major drawbacks are the huge number of tracks near the interaction point, which makes the search for prompt New Physics extremely challenging. Furthermore, the collision energy per nucleon is lower (e.g. $\sqrt{s_{\mathrm{PbPb}}}=5.02 \mathrm{TeV}$ ), which is however mostly problematic for heavy New Physics. Additionally, the LHC has allocated much less time to heavy ions runs than to protons runs. But most importantly the instantaneous luminosity is significantly lower for heavier ions.

The reason for the low luminosity is the strong electromagnetic field created in heavy ion collisions. It leads to new processes, namely Bound-Free Pair Production (BFPP) [3]

$$
{ }^{208} \mathrm{~Pb}^{82+}+{ }^{208} \mathrm{~Pb}^{82+} \stackrel{\gamma}{\rightarrow}{ }^{208} \mathrm{~Pb}^{82+}+{ }^{208} \mathrm{~Pb}^{81+}+e^{+},
$$

and Electromagnetic Dissociation (EMD) [4]

$$
{ }^{208} \mathrm{~Pb}^{82+}+{ }^{208} \mathrm{~Pb}^{82+} \stackrel{\gamma}{\rightarrow}{ }^{208} \mathrm{~Pb}^{82+}+{ }^{207} \mathrm{~Pb}^{82+}+n .
$$

\begin{tabular}{|c|c|c|c|c|c|c|c|c|c|c|c|c|}
\hline & \multirow[b]{2}{*}{$\begin{array}{c}M \\
{[\mathrm{GeV}]}\end{array}$} & \multirow[b]{2}{*}{$\begin{array}{l}\sqrt{s_{N N}} \\
{[\mathrm{TeV}]}\end{array}$} & \multicolumn{6}{|c|}{ Cross section } & \multicolumn{3}{|c|}{ Luminosity } & \multirow[b]{2}{*}{$\begin{array}{c}N_{\mathrm{N}} / N_{p} \\
\quad[1]\end{array}$} \\
\hline & & & $\begin{array}{c}\sigma_{\mathrm{EMD}} \\
{[\mathrm{b}]}\end{array}$ & $\begin{array}{c}\sigma_{\mathrm{BFPP}} \\
{[\mathrm{b}]}\end{array}$ & $\begin{array}{c}\sigma_{\text {had }} \\
{[\mathrm{b}]}\end{array}$ & $\begin{array}{c}\sigma_{\text {tot }} \\
{[\mathrm{b}]}\end{array}$ & $\begin{array}{l}\sigma_{W} \\
{[\mathrm{nb}]}\end{array}$ & $\begin{array}{c}A^{2} \sigma_{W} \\
{[\mu \mathrm{b}]}\end{array}$ & $\begin{array}{c}\mathscr{L}_{0} \\
{[1 / \mathrm{pbs}]}\end{array}$ & $\begin{array}{l}\tau_{b} \\
{[\mathrm{~h}]}\end{array}$ & $\begin{array}{c}\mathscr{L}_{\text {ave }} \\
{[1 / \mathrm{pbs}]}\end{array}$ & \\
\hline${ }_{1}^{1} \mathrm{H}$ & 0.931 & 14.0 & 0 & 0 & 0.071 & 0.071 & 20.3 & 0.0203 & $21 \cdot 10^{3}$ & 75.0 & $15.0 \cdot 10^{3}$ & 1 \\
\hline${ }_{8}^{16} \mathrm{O}$ & 14.9 & 7.00 & 0.074 & $24 \cdot 10^{-6}$ & 1.41 & 1.48 & 10.0 & 2.56 & 94.3 & 6.48 & 45.5 & 0.349 \\
\hline${ }_{18}^{40} \mathrm{Ar}$ & 37.3 & 6.30 & 1.2 & 0.0069 & 2.6 & 3.81 & 8.92 & 14.3 & 4.33 & 11.7 & 2.46 & 0.105 \\
\hline${ }_{20}^{40} \mathrm{Ca}$ & 37.3 & 7.00 & 1.6 & 0.014 & 2.6 & 4.21 & 10.0 & 16.0 & 2.90 & 12.9 & 1.69 & 0.0811 \\
\hline${ }_{36}^{78} \mathrm{Kr}$ & 72.7 & 6.46 & 12 & 0.88 & 4.06 & 16.9 & 9.16 & 55.7 & 0.311 & 9.80 & 0.169 & 0.0282 \\
\hline${ }_{36}^{84} \mathrm{Kr}$ & 78.2 & 6.00 & 13 & 0.88 & 4.26 & 18.1 & 8.43 & 59.5 & 0.311 & 9.15 & 0.166 & 0.0296 \\
\hline${ }_{54}^{129} \mathrm{Xe}$ & 120 & 5.86 & 52 & 15 & 5.67 & 72.7 & 8.22 & 137 & 0.0665 & 4.94 & 0.0294 & 0.0120 \\
\hline${ }_{82}^{208} \mathrm{~Pb}$ & 194 & 5.52 & 220 & 280 & 7.8 & 508 & 7.69 & 333 & 0.0136 & 1.57 & $3.8 \cdot 10^{-3}$ & 0.00379 \\
\hline
\end{tabular}

Table 1: Comparison of the cross section $\sigma$ and instantaneous Luminosity $\mathscr{L}$ for different ions. In the last columns we compare the number of produced $W$ bosons to the $p p$ LHC. 


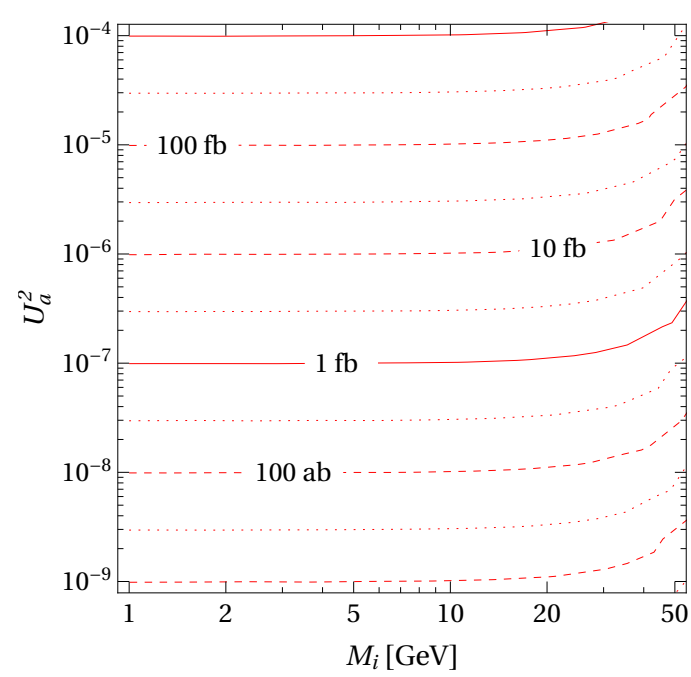

(a) Cross section of $p p \rightarrow W \rightarrow \ell N$.

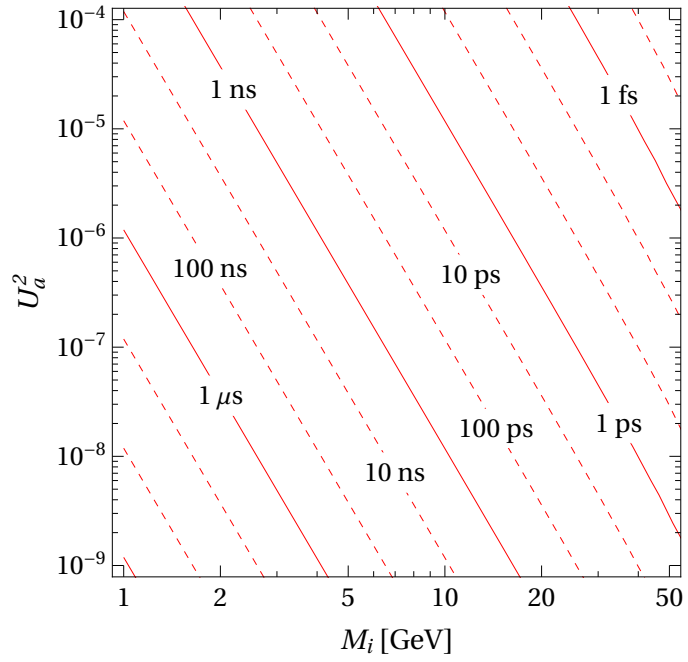

(b) Lifetime of HNLs

Figure 1: Cross section (Panel a) and lifetime (Panel b) of heavy neutrinos. In the relevant mass range the cross section is $\sigma \propto U_{a}^{-2}$ and masses of a few $\mathrm{GeV}$ lead to macroscopic displacement.

Both processes scale strongly with $A$ and $Z$

$$
\sigma_{\mathrm{EMD}} \propto \frac{(A-Z) Z^{3}}{A^{2 / 3}}, \quad \sigma_{\mathrm{BFPP}} \propto Z^{7} .
$$

This results in faster beam decay and the appearance of secondary beams, consisting of ions with different charge/mass ratio [5]. Although, it is possible to directed these secondary beams onto walls, extreme care has to be taken not to hit a magnet, as the energy deposition can accidentally cause a quench.

The instantaneous luminosity at one interaction point (IP) is $\mathscr{L} \propto N_{b}^{2}$ where $N_{b}$ are the number of ions per bunch [6]. The initial bunch intensity for arbitrary ions can be fitted to the known value of the lead run [7]

$$
N_{b}\left({ }_{Z}^{A} \mathrm{~N}\right)=N_{b}\left({ }_{82}^{208} \mathrm{~Pb}\right)\left(\frac{Z}{82}\right)^{-p}
$$

where $p=1$ corresponds to conservative and $p=1.9$ to optimistic assumptions. The loss of number of ions per bunch $N_{b}$ over time is given by the decay law

$$
\frac{\mathrm{d} N_{b}}{\mathrm{~d} t}=-\frac{N_{b}^{2}}{N_{0} \tau_{b}}, \quad \tau_{b}=\frac{n_{b}}{\sigma_{\mathrm{tot}} n_{\mathrm{IP}}} \frac{N_{0}}{\mathscr{L}_{0}},
$$

where $n_{\mathrm{IP}}$ is the number of interaction points. For a given turnaround time $t_{\text {ta }}$ between physics runs the integrated luminosity is maximised by $t_{\mathrm{opt}}=\tau_{b} \sqrt{\theta_{\mathrm{ta}}}$ with $\theta_{\mathrm{ta}}=t_{\mathrm{ta}} / \tau_{b}$ The average luminosity using the optimal run time is then

$$
\mathscr{L}_{\text {ave }}\left(t_{\text {opt }}\right)=\frac{L_{0}}{\left(1+\sqrt{\theta_{\mathrm{ta}}}\right)^{2}} .
$$




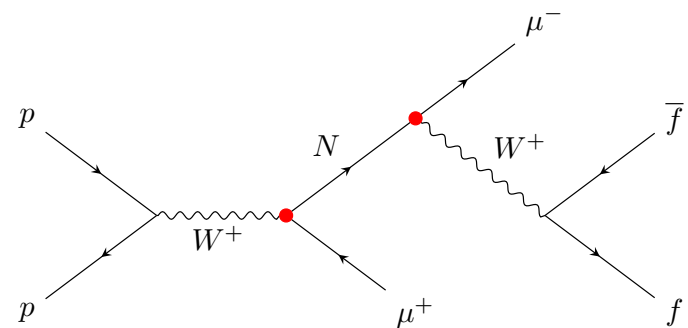

(a) $W$-induced HNL production.

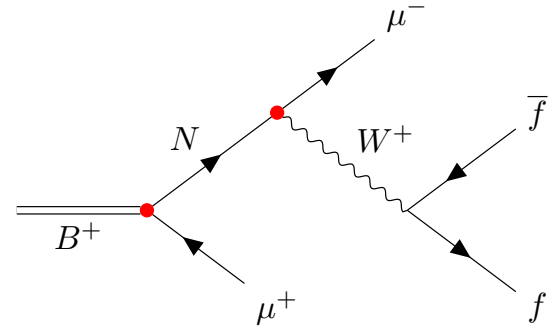

(b) B-induced HNL production.

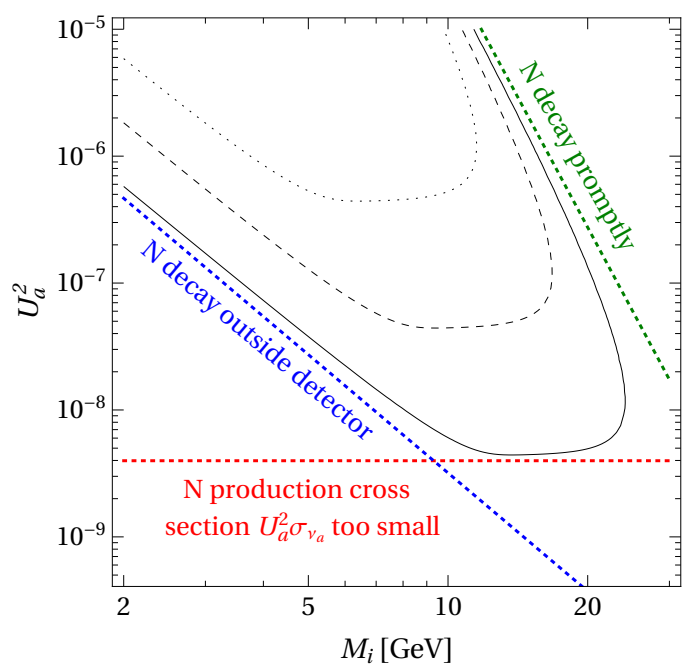

(c) Expected sensitivity in $p p$ collisions using $L=3,30,300 \mathrm{fb}^{-1}$.

Figure 2: Production processes (Panels a and b) and expected sensitivity using estimate (4.1) and major constraints (coloured) in Panel c.

These information suffice to estimate the number of produced particles in heavy ion collisions. We demonstrate this calculation for the production of $W$-bosons in Table 1. It demonstrates that the gain in cross section is overcompensated by the loss in luminosity. However, lower luminosity allows for very low triggers and lighter mediators are accessible.

\section{Heavy Neutral Leptons in the $\nu$ MSM}

In this analysis we use heavy neutrinos as an example for models with displace vertices. In the $\nu \mathrm{MSM}$ the $\mathrm{SM}$ is extended with three sterile neutrinos $\nu_{R i}[8]$

$$
\Delta \mathcal{L}=-y_{a i} \bar{\ell}_{a} \varepsilon \phi^{*} \nu_{R i}-\frac{1}{2} \overline{\nu_{R i}^{c}} M_{i} \nu_{R}+\text { h.c. }
$$

where $M_{i}$ is the Majorana mass matrix, which can be of order of the electroweak scale. After electroweak symmetry breaking the seesaw mechanism leads to three heavy mass eigenstates $N_{i} \simeq\left(\nu_{R}+\theta^{T} \nu_{L}^{c}\right)_{i}+$ c.c., where $\theta=v y M_{M}^{-1}$ and 3 light neutrinos $v_{i} \simeq V_{\nu}^{\dagger}\left(\nu_{L}-\theta \nu_{R}^{2}\right)_{i}+$ c.c. with a mass matrix $m_{\nu}=-\theta M_{M} \theta^{T}$. These parameter suffice to also explain the neutrino oscillation data. In the $\nu \mathrm{MSM}$ one of the neutrino decouples and becomes a dark matter candidate and another heavy neutrino can be a long lived state observable at the LHC (cf. Figure 1b).

\section{Heavy Neutral Leptons at the LHC}

We simulate $W$-boson mediated Heavy Neutral Leptons production (cf. Figures 1a and 2a) using MadGraph5_aMC@NLO [9, 10] which we have extended to be able to also simulate heavy ion collisions. In our analysis we trigger on the first $\mu$ with $p_{T}>25 \mathrm{GeV}$ and search 


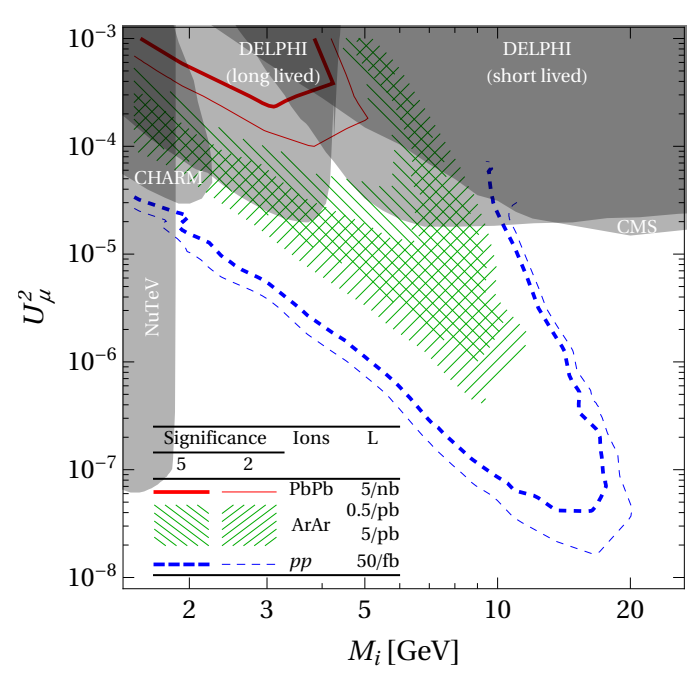

(a) Simulation for $W$-boson mediator

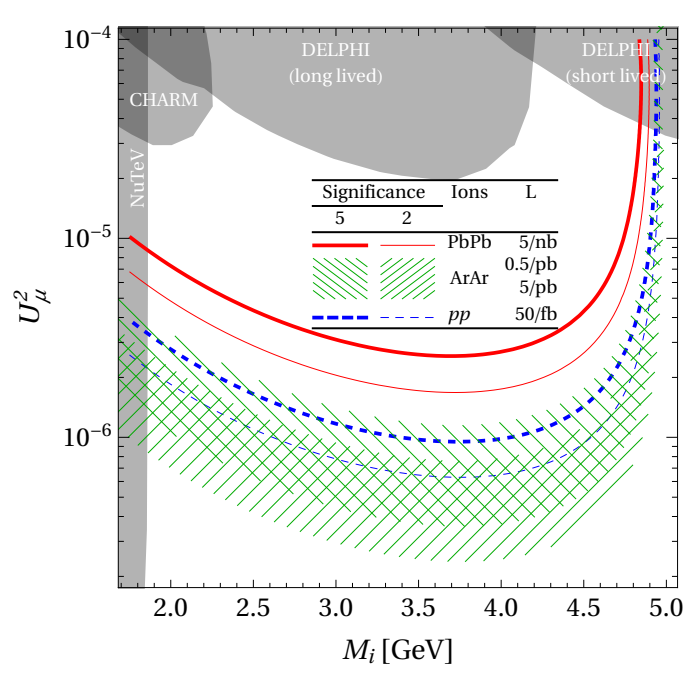

(b) Estimate for $B$-meson mediator

Figure 3: Comparison of the sensitivity for heavy neutral leptons in $p p, \mathrm{Ar} \mathrm{Ar}$, and $\mathrm{PbPb}$ collisions for $W$-boson (Panel a) and B-meson (Panel b) induced processes. All event numbers are given for one month run time.

for displaced $\mu$ with $d>5 \mathrm{~mm}$. For $p p$ collisions this search strategy has been studied in great detail [11]. The number of events that can be seen in a detector can be estimated as

$$
N_{d} \sim L_{\text {int }} \sigma_{\nu} U^{2}\left(e^{-l_{0} / \lambda_{N}}-e^{-l_{1} / \lambda_{N}}\right) f_{\text {cut }}
$$

here $l_{1}$ is the length of the effective detector volume and $l_{0}$ the minimal displacement that is required by the trigger and we use $l_{0}=5 \mathrm{~mm}$ and $l_{1}=20 \mathrm{~cm} . \lambda_{N}=\beta \gamma / \Gamma_{N}$ is the decay length of the heavy neutrino and $f_{\text {cut }}$ contains all efficiencies. The decay rate can be estimated to be

$$
\Gamma_{N} \simeq 11.9 \cdot \frac{G_{F}^{2}}{96 \pi^{3}} U^{2} M^{5}
$$

The result of the estimate (4.1) is shown in Figure 2c. For $B$-meson mediated processes shown in Figure $2 \mathrm{~b}$, lower trigger thresholds are beneficial and in the case of heavy ion collisions we use $p_{T}>3 \mathrm{GeV}$. This strategy is already probed at LHCb and also considered by CMS using parked data. The modification of estimate (4.1) taking the allowed phase space into account is

$$
N_{d}=\frac{L_{\mathrm{int}} \sigma_{B}^{[A, Z]}}{9}\left[1-\left(\frac{M_{i}}{m_{B}}\right)^{2}\right]^{2} \cdot U^{2}\left(e^{-l_{0} / \lambda_{N}}-e^{-l_{1} / \lambda_{N}}\right) f_{\text {cut }},
$$

leading to a sharp cutoff at the $B$-meson mass.

\section{Results and Conclusion}

In Figure 3 we show the results of our analysis for $W$-boson induced processes and of the analytic estimate for $B$-meson induced processes, both for $p p$, $\mathrm{ArAr}$, and $\mathrm{PbPb}$ 
collisions under the assumption of equal running time. $B$-meson induced processes profit substantially from the lower trigger requirements in heavy ion collisions.

We have shown that heavy ion collisions allow to search for New Physics with displaced signatures, as such searches circumvent the busy inner tracker. We point out that the lower trigger requirements could be the key advantage of heavy ion collisions over proton collisions and that intermediate ions can be very interesting for searches of New Physics. We have demonstrated this idea using the example of heavy neutrinos, but expect it to be just as well applicable to other models with displaced signatures.

\section{References}

[1] M. Drewes et al. "A Heavy Metal Path to New Physics" (2018). arXiv: 1810.09400 [hep-ph].

[2] M. Drewes et al. "Long Lived Particles Searches in Heavy Ion Collisions at the LHC" (2019). arXiv: 1905.09828 [hep-ph].

[3] H. Meier et al. "Bound free electron positron pair production in relativistic heavy ion collisions". Phys. Rev. A63 (2001), p. 032713. DoI: 10.1103/PhysRevA.63.032713. arXiv: nucl-th/0008020 [nucl-th].

[4] I. A. Pshenichnov et al. "Mutual heavy ion dissociation in peripheral collisions at ultrarelativistic energies". Phys. Rev. C64 (2001), p. 024903. DOI: 10.1103/PhysRevC. 64.024903. arXiv: nucl-th/0101035 [nucl-th].

[5] M. Schaumann. "Heavy-ion performance of the LHC and future colliders". PhD thesis. Aachen, Germany: RWTH Aachen U., 2015.

[6] M. Benedikt, D. Schulte, and F. Zimmermann. "Optimizing integrated luminosity of future hadron colliders". Phys. Rev. ST Accel. Beams 18 (2015), p. 101002. DoI: 10.1103/PhysRevSTAB.18.101002.

[7] J. Jowett. "HL-LHC performance: Update for HE-LHC and light ions" (2018). URL: https://indico.cern.ch/event/686494/timetable.

[8] T. Asaka and M. Shaposhnikov. "The $\nu$ MSM, dark matter and baryon asymmetry of the universe". Phys. Lett. B620 (2005), pp. 17-26. DOI: 10.1016/j ·physletb. 2005. 06.020. arXiv: hep-ph/0505013 [hep-ph].

[9] J. Alwall et al. "MadGraph 5: Going Beyond". JHEP 06 (2011), p. 128. DOI: 10. 1007/JHEP06 (2011) 128. arXiv: 1106.0522 [hep-ph].

[10] C. Degrande et al. "Fully-Automated Precision Predictions for Heavy Neutrino Production Mechanisms at Hadron Colliders". Phys. Rev. D94.5 (2016), p. 053002. DOI: 10.1103/PhysRevD.94.053002. arXiv: 1602.06957 [hep-ph].

[11] M. Drewes and J. Hajer. "Heavy Neutrinos in displaced vertex searches at the LHC and HL-LHC" (2019). arXiv: 1903.06100 [hep-ph]. 